\title{
46th ANNUAL SASKATCHEWAN CHRISTMAS BIRD COUNT - 1987
}

Compiled by MARY I. HOUSTON, 863 University Drive, Saskatoon, Saskatchewan. S7N 0J8

Saskatchewan began its second Century of Concern for Wildlife with an all-time record of 611 Christmas bird counters in a near-record 82 localities (83 in 1986). Such increased participation, aided by mild weather, produced more variety than ever. The number of species observed throughout the province far exceeded previous totals -97 species (plus one race) were seen on count days, with two additional species during count period for a total of 99 species recorded between 17 December and 3 January. The previous high was 84 species (78 seen on count days with 6 additionals during count period) in 1981.

Some centers responded with enthusiasm to the "kick-on" theme, master. minded by Cam and Joy Finley in Edmonton. Prince Albert National Park outdid all other Canadian centers in terms of the greatest per capita participation in Canada, since 28 of its 48 participants were residents or staff at Waskesiu which has a winter (off-season) population of only 90. (Last year they had 10 observers out). They also sighted a record number of species (21) and individuals (435) for their area on a count day.

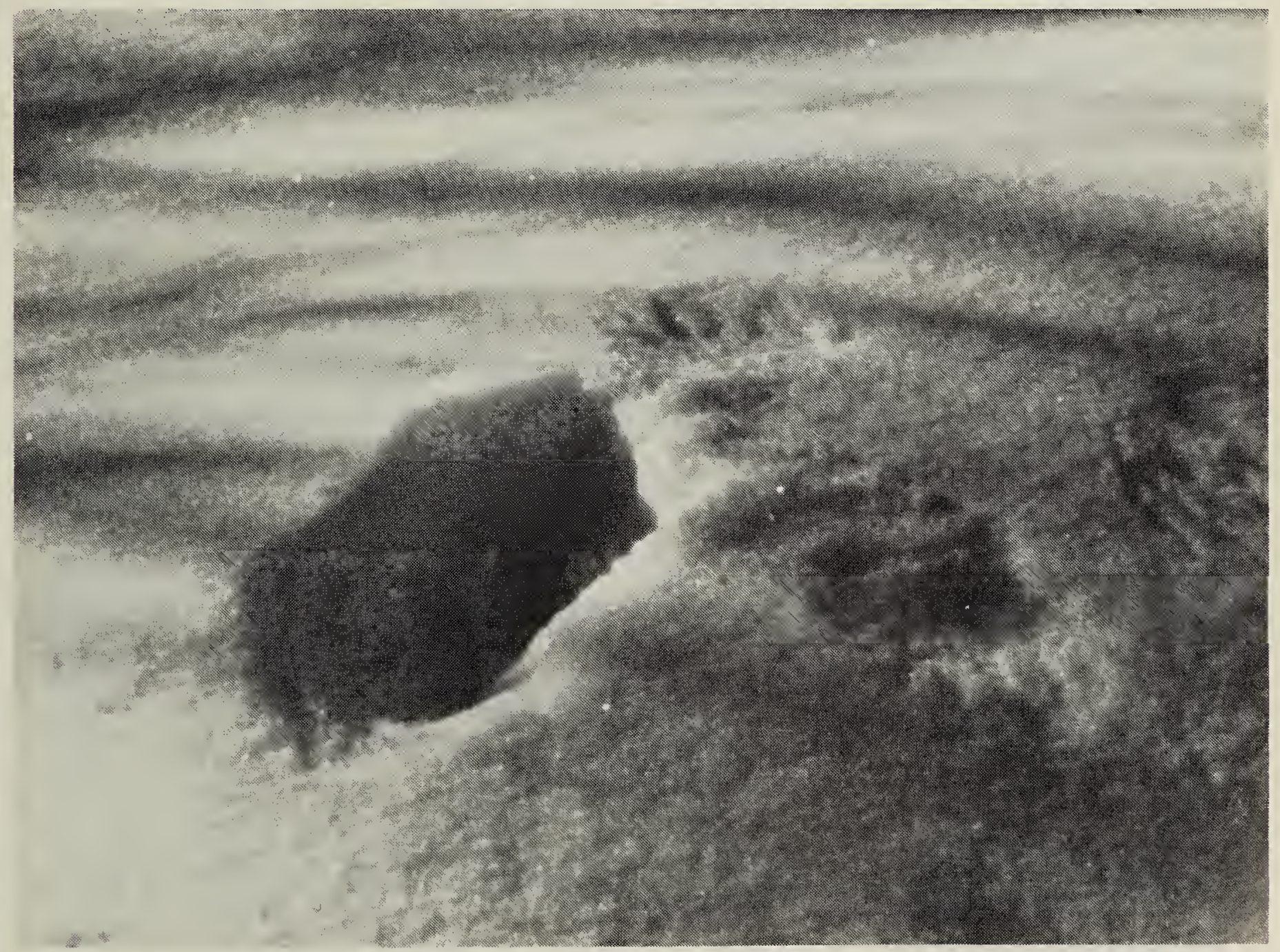


Similarly, Duval increased participation from 4 last year to 9 this year; Fort Walsh, from 14 to 19; Govenlock, 10 to 14 ; Indian Head, 34 to 41; Kelvington, 2 to 9; Moose Jaw, 26 to 30; Pike Lake, 12 to 18; Regina, 35 to 44; Saskatoon, 50 to 74; and Weyburn, 10 to 21 . Weather conditions and ease of travel were equally favourable in 1986, so congratulations are due to all those who made the special effort to "get involved" this year.

The only new species added to the all time list was a Water Pipit seen at Govenlock. This brings the species seen on Count Days to 144, with 5 additional species seen during count periods, for an all-time total of 149 Christmas period species.

The other exciting event was a minor incursion of Mountain Chickadees. Single individuals were reported from Fort Qu'Appelle, Marsden and Skull Creek. The only previous $C B C$ record was from Skull Creek in 1966. Another noteworthy sighting was of a Rosy Finch at Loon Lake, north of its usual winter range, from 1 through 20 December. More summer birds than usual remained over Christmas, probably because of the very mild weather. Frank Roy reported that only once before in all recorded history, in 1974, had Cold Lake been still completely ice free by 25 December. In addition to various land birds lingering on, the waterbirds included a Tundra Swan (2 localities), Double-crested Cormorant (2 localities), 8 species of ducks and 5 species of gulls. On the other hand, numbers of Grosbeaks, Redpolls, Snow Buntings and Waxwings were low in most localities.

At Naicam, Ron and Julie Jensen reported a large, white gull which best fit the description of a Glaucous-winged Gull, never before reported in Saskatchewan. However, since there were no substantiating photographs available, we have listed it as an unidentified gull. (For details, see article in this issue).

Following is a list of participants from the 82 localities and a map. Four tables then summarize the results. Table 1 provides the coverage and weather. Table 2 lists the species reported from more than five localities on count day, indicating additional species during count period by means of a + sign. Table 3 gives numbers and locations for species seen in five or fewer locations. Table 4 present numbers and locations of those birds unidentified as to species.

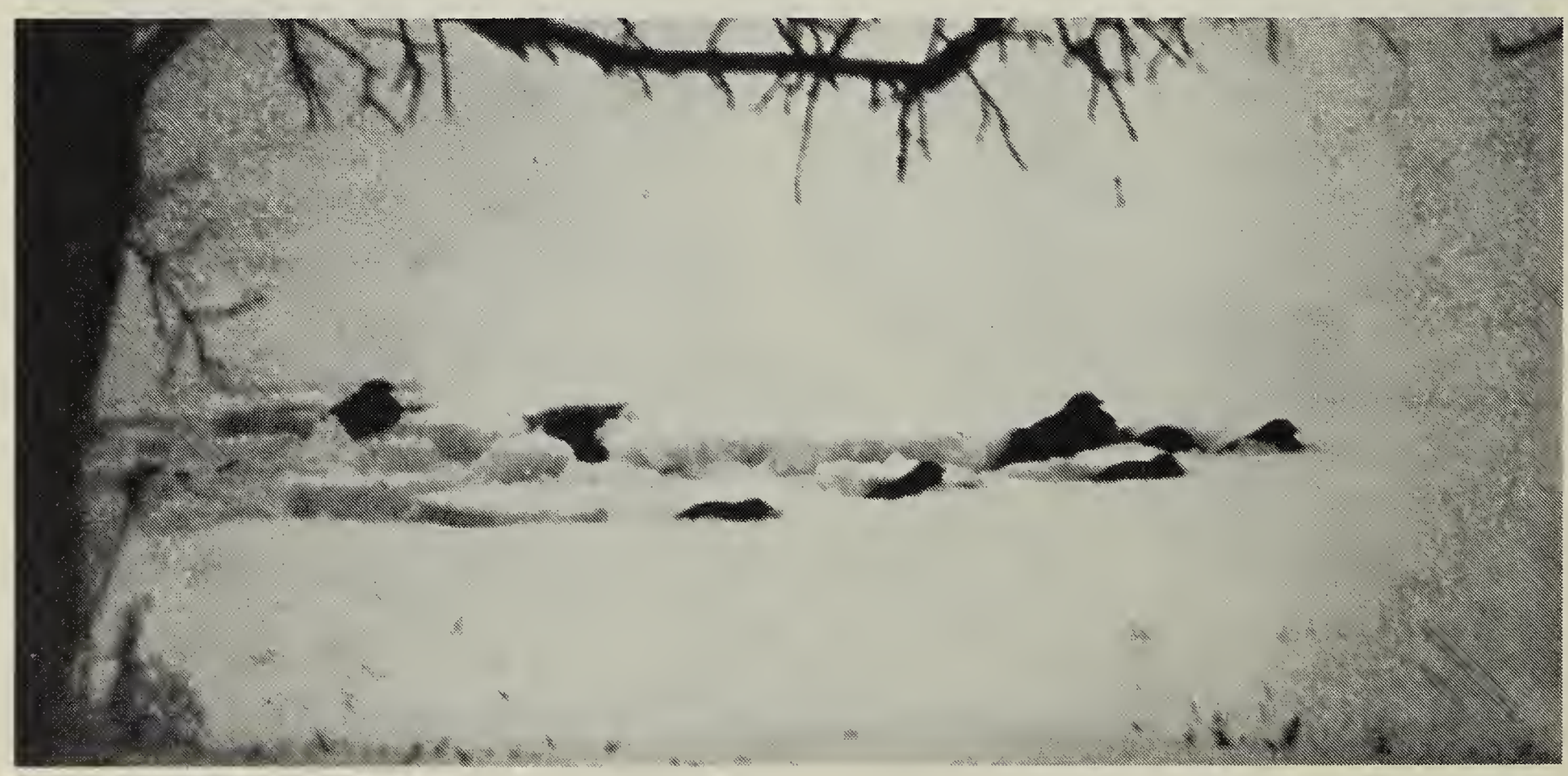




\section{Count Areas and Participants}

(Names of compilers are in italics. Numbers of participants in each count are in parentheses following the names.)

1. ABERNETHY-KATEPWA. Isabel Barnsley, Dick Barton, Ron Bittner, Del Cairns, Jake Cairns, Marge Chomoway, Ron Hooper, Alice Laing, Joy McKen, Lorne Rowell, Wanda Stueck. (11)

2. ASSINIBOIA. Ed Bearss, Gordon Brunt, Jack Burgeson, Cecil $T$. Hayward, Wilf Prentice, Ken Schuweiler. (6)

3. BANGOR. Jean Hilton, Sheila Popowich, Don Thompson. (3)

4. BATTLEFORD. Jean Anderson, Barry Craig, Ron Dewald. (3)

5. BIGGAR. Arvid Pederson, Guy Wapple, Marguerite Wapple, Robert Wapple, Rodney Wapple. (5)

6. BIG GULLY CREEK. Anemone Harris, Valeriana Harris, Wayne Harris, Sheila Lamont. (4)

7. BIRCH HILLS. Moe Mareschal, Terry Toews, Don Weidl. (3)

8. BROADVIEW. Dave J. Chaskavich, Don Weidl. (2)

9. BROMHEAD. Carol Bjorklund, Wayne Harris. (2)

10. CABRI. Carman Dodge, Genevieve Greif. (2)

11. CARRAGANA. Margaret Back, Stan Back. (2)

12. CLEARWATER LAKE. Cliff Matthews. (1)

13. CODETTE. W.G. Harstad. (1)

14. CRAVEN. Samuel Bergerman, Curtis Buhay. (2)

15. CROOKED LAKE P.P. Doug Boivin, David Chaskavich, Don Weidl, Tony Weidl. (4)

16. DALMENY. Jeremy Quiring, Stu Quiring, Karen Rispin, Loyd Sperling. (4)

17. DILKE. Margaret Belcher, Ken McArton, George Metz, Bertha Wilson. (4)

18. DUVAL. Rolf Antonowitsch, Wendy Antonowitsch, Enid E. Cumming, George Herber, Joe Materi, Dean J.
Nernberg, Lloyd S. Saul, Mark Todd, Lyle Turner. (9)

19. ENDEAVOUR. Norman Harris. (1)

20. ESTEVAN. Alan R. Smith. (1)

21. FORT QU'APPELLE. Dick Barton, Del Cairns, Rus Cairns, Bernard De Vries, Ronald Hooper, Alice Laing, Lois Lamontagne, Vic Lamontagne, Jack Lowe, Allan Mlazgar, Raymond Mlazgar, Paul Paquin, Mary Pletz, Enola Rak, Harold Ross, Doreen Rowell, Lorne Rowell, Joe Rumancik, Steve Rumancik, Ron Simmonds, Mary Jo Skolensky, Dorothy Williams. (22)

22. FORT WALSH. David Bailey, Martin Bailey, Carol Bjorklund, Dixie Black, Paul Chytyk, Enid Cumming, Bob Ewart, Anemone Harris, Valeriana Harris, Wayne Harris, Trevor Herriott, Paul James, Bob Kreba, Sheila Lamont, Susan McAdam, Ron Meyers, Wilkes Parsonage, Sandra Wapple, Guy Wapple. (19)

23. GARDINER DAM. Harley Forsberg, Anemone Harris, Valeriana Harris, Wayne Harris, Sheila Lamont, Susan McAdam, Guy Wapple, Sandra Wapple. (7)

24. GLAMIS-WISETON. Grev L. Jones. (1) 25. GOOD SPIRIT LAKE. Bill Anaka, Joyce Anaka, Julia Wiwchar. (3)

26. GOVENLOCK. David Bailey, Martin Bailey, Carol Bjorklund, Paul Chytyk, Enid Cumming, Anemone Harris, Valeriana Harris, Wayne Harris, Robert Kreba, Sheila Lamont, Susan McAdam, Ron Myers, Guy Wapple, Sandra Wapple. (14)

27. GRAND CENTRE-PIERCELAND. Elaine Goh, Bill Kervin, Frank Roy, Margaret Roy. (4)

28. GREENWATER LAKE PARK. Jean Dalke, Herman Duerksen, Don Forbes, Donald Hooper, Leila Hooper, Doris Terry. (6)

29. HERBERT. Don Weidl, Brenda Winch. (2)

30. HUMBOLDT. Ed Brockmeyer, Mike Volk. (2) 
31. INDIAN HEAD. Mr. and Mrs. Peter Barrett, Mrs. Jan Beattie, Corol, Coralee, Denise, Graeme and Mark Beaulieu, Vic Beaulieu, Bernard and Marg Bosgoed, Yvonne Brown, Eric, Kara and Roger Gray, Gordon Howe, Helen King, Greta, John, Mary, Peter and Winda Kort, Rose and Roy McLaughlin, Bruce, Claire, Erin and Richard Neill, Dora Nichols, Adam, Heidi, Joan and Lorne Scott, Joyce Skinner, Mary Skinner, Gordon Willerth, Garbara, Garth Jackie, Ken, Yocelyn Willoughby. (41)

32. KAMSACK-TOGO. Mabel Bausiuk, Jean Bobick, Fred Erhart, Anita Klucho, Cusie May, Archie MacDonald, Ann Mclvor, Jessie and Dallas Rezansoff, Isabel Ritchie, Stan Stone, Sr., Grace Theis. (12)

33. KEDLESTON (east of Dilke). Doug Laing, Vera Laing, George Metz (3)

34. KELVINGTON. Patrick Finnie, Helen Hilbig, Howard Hope, Ruth Hope, Jill Rennie, Lloyd Saul, Dianne Sloan, Graham Sloan, Marguerite Sloan. (9)

35. KENASTON. Lawrence Beckie. (1)

36. KINDERSLEY. Gerry Essar, Michael Essar. (2)

37. KUTAWAGAN LAKE. Wayne Harris, Sheila Lamont. (2)

38. LA RONGE. Jim Paul. (1)

39. LAST MOUNTAIN LAKE WILDLIFE MANAGEMENT UNIT. Anemone Harris, Valeriana Harris, Wayne Harris, Sheila Lamont (4)

40. LEADER. Daisy D. Meyers. (1)

41. LEASK. Alvin Lasich. (1)

42. LITTLE MANITOU LAKE. E. A. Driver, Alan R. Smith. (2)

43. LIVELONG. Sarah Pavka. (1)

44. LOON LAKE. Penny Davis, Frank Scott, Paul Scott. (3)

45. LOVE-TORCH RIVER. Bert Dalziel, Duke Dalziel, Joan Dalziel, Betty Donovan, Bruce Donovan, Dorothy Humphrey, Bill Matthews, Lynn Matthews, Dave Sundberg, Irene Westman, Stan Westman. (11)
46. LUSELAND. Bob and Val Finley, Estelle Finley, Kim Finley, Bill Frey, Bill and Donna Holton, Bev, Brent, Dustin and Levi Honeker, Katrina and Travis Martfeld, Art and Lois Meier, Joe Roszlein, Ev Wagner. (17)

47. MAIDSTONE BRIDGE. Anemone Harris, Valeriana Harris, Wayne Harris, Dan Lamont, Sheila Lamont. (5)

48. MARSDEN. Eileen Graham, Lois $A$. Wooding. (2)

49. MAYMONT. Donovan G. Florence. (1)

50. MELVILLE. Marion MacLean. (1)

51. MISSINIPE. Shirley Glass. (1)

52. MOOSE JAW. Fran and Jim Bacon, Orville Barnstable, Edith Bell, Doug and Helen Brunsden, Ben and Isabelle Cruise, John Docherty, Doug Francis, Ken Guy, Kerry Hanley, Pat Kern, Eve King, Cy Knight, Leith Knight, Connie and Hugh Mclntyre, Richard and Wilma Pickering, Gavina Reekie, Hubert Ripley, Arie and Henry Van Dorland, Sheina Wait, Ed and Grace Walker, Harvey and Maureen Williams, Casey Zath. (30)

53. NAICAM. Julie Jensen, Ron Jensen, W. Yanchinski. (3)

54. NIPAWIN. Inge Brown, Betty Christiansen, Joyce Christiansen, W.G. Harstad. (4)

55. PIKE LAKE. Paul and Vi Coutu, Marie Dunn, Hartley Fredeen, Mary Gilliland, Bernie Gollop, Helen Hobbs, Ron Jensen, Gwenna Moss, Menno Nickel, Evelyn Paterson, Frank Roy, Lorne Sperling, Nan Story, Ivor Thokle, Jim Wedgwood, Brock and Kay Whale. (18)

56. PINE CREE REGIONAL PARK (NE of Eastend). James Donovan. (1)

57. PREECEVILLE. Raza Bezan, James $H$. Purdy. (2)

58. PRINCE ALBERT E. Christie Aschim, Pamela Burt. (2)

59. PRINCE ALBERT CITY. Carman Dodge, Genevieve Greif, Robert Luterbach, Ted Snow. (4)

60. PRINCE ALBERT NATIONAL PARK 
(PANP). Marilyn Anions, Monte Anions, Eve Attiken, Matthew Awbry, Peter Bakker, Erin Baydack, Karen Baydack, Joey Beaudry, Devon Bell, Doug, Faye, Lee and Meredith Burles, Shawn Cardiff, Shane Carlson, Susan Carr, Dave and Josh Conard, Jan, Peter and Sean Garden, Jason Hargrave, Jim Helm, Doug Hodgins, Arnold Janzen, Darlene and Peter Krasko, Gail and Howard Lancaster, Bob Luterbach, Richard Marr, Brent McDougall, Eugene McNabb, Irene Mooney, Brad Muir, Laura Muir, Sharon Putz, Chris Regal, Becky and Eike Scheffler, Jessie Sinclair, Petra Sjouwerman, Merv Syroteuk, Bob Turnbull, Donna, Richard and Ron Umpherville, Joy Unger. (48)

61. QU'APPELLE DAM. Christopher Honig, Glenn Honig. (2)

62. RAYMORE. Anemone Harris, Valeriana Harris, Wayne Harris, Sheila Lamont. (4)

63. REGINA. Martin Bailey, Keith Barr, Margaret Belcher, Inez Benesh, Sasha Bernatsky, Tom Beveridge, Carol Bjorklund, Eric Cooke, Kirsten Costain Jim Elliott, Bob and Sandra Ewart, Kay Ferguson, Margot Gilligan, Janet Harmsworth, Karen, Katie and Trevor Herriot, Norm Herriot, Dale and Paule Hjertaas, Waren Hjertaas, Phyllis Ilsley, Lillian Jones, Robert Kreba, Carol Kujala, Tony Lang, Lynda Langenbacher, Christine MacDonald, John MacDonald, Lauren Mang, Miles Myers, Dean Nernberg, Brian Rainey, Gillian and Trevor Richardson, Dean and Miriam Richert, Tom Riffel, Jim Rydzik, Pat Sargent, Diane Secoy, Franik and lan Switzer, Jon Triffo. (44) 64. ROUND L.AKE. Doug Francis, Joe Grimeau, Pat Kern, Leo Tremblay. (4)

65. SASKATOON. Norma Allen, Juhachi and Mika Asai, Keith Berg, Bob, Garth, Joyce Besant, Maxine Birnie, Ray Bisha, John Bond, Phyllis Brown, Cynthia Campbell, Henry Cariou, Muriel Carlson, Ruby Clarke,
Sabine Cordes, Paul and Vi Coutu, Jean Cowell, J. Gifford, Michael Crane, Alan, Arthur, Hartley, Katherine, Kenneth and Linda Fredeen, Eric Densem, Marie Gillespie, Mary Gilliland, Bernard, M.F. and Michael Gollop, Fred Goodliffe, Jackie Graham, Bob Green, Bruce Hanbidge, Marty Helgerson, R. W. Hogg, Jane Houlden, David, Don, Mary and Stuart Houston, Bob and Suzy Kohlmeier, Janis Laliberte, Blake and Kay Maybank, Audrey McKibbon, Don and Jo McRobbie, David and Quentin Miller, Betty Mundy, Menno Nickel, Muriel Park, Evelyn Paterson, Donna Pletz, Wilf Ready, David Richeson, Alvena Schnell, Elizabeth Skopik, Jim and Judy Slimmon, Phil Taylor, Ivor Thokle, Jeff Turple, Lena Vella, Hilda Voth, Heather Wagg, Jim Wedgwood, Jim Wood, David Wright. (74)

66. SCOTT. Guy Wapple, Sandra Wapple. (2)

67. SKULL CREEK. Allan Bennetto, James Bennetto, Doug, Marjorie and Robert Mann, Patty and Peter Robertshaw, Marina and Ray Schuler. (9)

68. SNOWDEN. Edward, Elodie, Irene and Karen Hagel, Danelle, Deni, Stacii and Verna Messer, lain Richardson, Lloyd S. Saul, Lyle Turner. (11)

69. SOMME. Edwin and Shirley Billeter, Sharon Birch, David Black, Florence Chase, Donald Hooper, Gordon Johnston. (7)

70. SPINNEY HILL (near Baljennie). E. $A$. Driver, Paul Stevens. (2)

71. SPRING VALLEY. Flossie Bogdan, Nick Bogdan. (2)

72. SQUAW RAPIDS. Chris Adam, Anemone Harris, Wayne Harris, Sheila Lamont, Susan McAdam, Stan Shadick, Guy Wapple, Sandra Wapple. (8)

73. TISDALE. Joyce Mohr. (1)

74. TOMPKINS. Raymond Olson. (1)

75. TUBEROSE. Cliff Matthews, Gordon and Steven Moreside. (3) 
76. WARMAN. Murray Doell. (1)

77. WEYBURN. Bill Aldrich, Leo Belanger, Louise Belanger, Ray Belanger, Sophie Belanger, Greg Bobbitt, Jim Charlton, Chris Douglas, Ross Douglas, Norm Flaten, Dick Gutfriend, Dorothy Hannah, Grace Kurtz, Betty Layh, Phil Layh, Cyril Marcotte, Ray Neville, Wally Nicholson, Nick Postey, Doug Pulfer, Stewart Stairmand. (21)

78. WHITE BEAR. Darryl, Floyd, Gary and Laine Jordheim, Sig Jordheim, Greg McCullock. (6)

79. WHITE BEAR LAKE (North of Carlyle).
D. G. Bobbit, Ross Douglas. Wally Nicholson, Nick Postey, Stewart Stairmand. (5)

80. WHITEBEECH (North of Arran). Ida Wotherspoon, Lindsay Wotherspoon. (2)

81. WOLSELEY. Dale Chay, Donald Hayward. (2)

82. YORKTON. Bill and Joyce Anaka, Joyce Baines, Joe Bergerman, Art Griffith, Elenor and Warren Hjertaas, Lance Irvine, Jim Jowsey, Lorne and Marilyn Lepp, Bret Parlee, Ed and Dorothy Skeene, Beth Turberfield, Harold Wilkinson, Julia Wiwchar. (17)

\section{GLAUCOUS-WINGED GULL AT NAICAM}

RON JENSEN, 1027 King Crescent, Saskatoon, Saskatchewan. S7K ON9

The Christmas Bird Count at Naicam on 19 December produced the usual hardy winter birds - and one unusual exception, a Glaucous-winged Gull. This large white-headed gull was feeding on the grid road about $3 \mathrm{~km}$ west of Naicam. From a distance of $50 \mathrm{~m}$ the white head, breast, belly and tail, grey back and wings, pink feet and yellow bill were distinctly seen. Within seconds, a vehicle passed, putting the bird to flight.

Between $12: 15$ and $12: 25$, using $8 \times 30$ binoculars and with Birds of North America by Robbins et al as a reference, the gull was viewed in flight and then at rest on a stubble field. ${ }^{6}$ Viewing conditions were good; although partially overcast it was bright, with light reflecting from the fresh-fallen snow. As the gull flew in arching circles within 150 $\mathrm{m}$, the colour of the primaries were observed to be the same grey that mantled the back and wings. This was the best feature for separating the Glaucous-winged from the Glaucous Gull as depicted by Robbins. When it settled again within $75 \mathrm{~m}$ its large size was noted, roughly equal to that of a Herring or California Gull, with which the author is familiar. The large size, with the tail as long as or longer than the wing tips, excluded the smaller Iceland Gull. Once more the pink feet, yellow bill with a red dot, and a yellow eye were noted, all of which matched the text and illustration in Robbins for the Glaucous-winged Gull. ${ }^{6}$

Julie Jensen and the author returned at 13:30 with a camera. The gull was uncooperative, flying and resting beyond camera range. Blowing snow made viewing conditions less satisfactory. We 